\section{A relação entre estrutura ocupacional e acesso a plano de saúde no Brasil: uma análise para 1998 e 2003}

\author{
Labor market structure and access to private \\ health insurance in Brazil
}

\author{
1 Faculdade de Ciências \\ Econômicas, Universidade \\ Federal de Minas Gerais, \\ Belo Horizonte, Brasil. \\ Correspondência \\ M. V. Andrade \\ Centro de Desenvolvimento \\ e Planejamento Regional, \\ Faculdade de Ciências \\ Econômicas, Universidade \\ Federal de Minas Gerais. \\ Av. Antonio Carlos 6627, \\ Belo Horizonte, $M G$ \\ 30170-120, Brasil. \\ mviegas@cedeplar.ufmg.br
}

\begin{abstract}
This paper aims to describe health insurance coverage among different types of workers in Brazil. Health insurance coverage and labor market insertion are used to define homogeneous groups of workers. The Grade of Membership method is used to build a typology of workers. The database was the Brazilian National Household Survey (PNAD) for 1998 and 2003, including a health survey. Five worker profiles were defined. The key variables were: health insurance coverage, schooling, and work status. The main findings show a positive association between health insurance coverage, income from work, and trade union membership.
\end{abstract}

Prepaid Health Plans; Private Health Care Coverage; Job Market
Ana Flavia Machado 1

Mônica Viegas Andrade 1

Ana Carolina Maia 1

\section{Introdução}

Um aspecto presente na literatura de economia da saúde diz respeito aos impactos da presença de planos de saúde no mercado de trabalho. Os planos de saúde são benefícios indiretos aos trabalhadores e representam um custo fixo elevado para os empregadores, determinando uma maior rigidez salarial e alterando, para os empregadores, a estrutura de oferta de emprego e, para os empregados, a escolha de postos de trabalho.

A análise de diferenciais de salários e de rigidez salarial tem seu início com as teorias duais do mercado de trabalho que buscavam explicar a segmentação do mercado de trabalho. Nos anos 80, a discussão sobre segmentação é retomada pelos teóricos dos modelos de salário-eficiência 1,2,3,4, que por meio das hipóteses de informação imperfeita e de uma função esforço-salário, buscam demonstrar a racionalidade das firmas que pagam salários acima daquele que asseguraria o pleno-emprego. Os resultados obtidos evidenciam que as firmas são bem sucedidas nessa estratégia, porque reduzem a mobilidade de mão de obra, ampliando a produtividade do trabalho.

Em que pese o aumento dos custos trabalhistas advindos de elevados salários diretos e/ ou existência de benefícios, as firmas se orientam por uma racionalidade econômica que é justamente o aumento da motivação do trabalhador e, consequentemente, da produtividade 
do trabalho. Se considerarmos o caso específico do acesso a planos de saúde, esse retorno pode ser ainda maior porque os trabalhadores podem cuidar do seu estado de saúde, por meio de exames clínicos rotineiros, o que contribui para assiduidade e melhor desempenho nas atividades laborais.

Recentemente, a literatura sobre economia da saúde em países desenvolvidos tem se preocupado com a relação entre acesso a plano de saúde e mobilidade no mercado de trabalho 5,6,7. Nos Estados Unidos, cerca de $90 \%$ da população coberta por planos de saúde tem acesso a plano de saúde por intermédio do emprego ${ }^{6}$. A provisão de seguro de saúde é baseada em decisões privadas dos trabalhadores e das firmas, e isso tende a reduzir a mobilidade uma que vez aprisiona os trabalhadores, receosos de perder tal benefício, mesmo surgindo oportunidade em postos de trabalho com elevada produtividade e, portanto, salários mais elevados. Em razão dessa racionalidade, a estratégia foi denominada job lock.

Ademais, o aumento dos prêmios de segurosaúde pode provocar mudanças na contratação de trabalho, tendo efeitos tanto na oferta quanto na demanda por horas ${ }^{6}$. Um novo emprego pode significar não cobertura de planos ou exclusões de condições preexistentes, além da exigência de períodos probatórios para nova cobertura. O posto de trabalho atual pode oferecer uma variedade de opções de seguro que não está disponível nos outros postos que também oferecem seguro, tornando a mudança pouco atrativa.

Por último, cabe mencionar que as características individuais e os arranjos familiares dos trabalhadores afetam suas preferências e têm impactos tanto na escolha de inserção no mercado de trabalho quanto na escolha de acesso a plano de saúde. Assim sendo, a relação entre acesso a plano de saúde e a inserção no mercado de trabalho evidencia que, para além da organização dos mercados produtivos e de trabalho, os atributos dos indivíduos, tais como experiência, idade e escolaridade, interferem nessa relação. Este trabalho investiga a relação entre inserção no mercado de trabalho, características individuais e posse de plano de saúde mediante a construção de uma tipologia.

No Brasil o sistema de saúde é misto, existindo uma complementaridade entre o setor público e o setor de saúde suplementar. Embora a Constituição Federal de 1988 tenha garantido o acesso universal a todos os níveis de atenção médico-hospitalar pelo Sistema Único de Saúde (SUS), uma parcela da população, 25\%, prefere recorrer ao sistema de saúde suplementar. Assim como nos Estados Unidos, no Brasil, o mercado de trabalho também é uma porta de entrada importante para o setor de saúde suplementar: em 2003 , cerca de $65 \%$ da população com cobertura privada teve acesso aos planos de saúde por meio do vínculo empregatício ${ }^{8}$.

Uma das razões para a ainda baixa cobertura de planos e seguros de saúde observada no Brasil é a natureza segmentada do mercado de trabalho. Sem considerar a complexidade dos arranjos e relações econômicas, podemos classificá-lo em três estruturas: uma mais formalizada com emprego de tecnologia mais avançada, em que são mais nítidas as características de mercados internos de trabalho; a segunda compreenderia empresas registradas com emprego de mão de obra assalariada atuando em mercados mais concorrenciais; e, por fim, um expressivo contingente da força de trabalho, cerca de $50 \%$ dos ocupados que se inserem no mercado de trabalho informal, como assalariados sem carteira e trabalhadores por conta própria. No caso dos dois últimos grandes segmentos, dificilmente o trabalhador tem acesso a plano de saúde coletivo e poucos são aqueles com planos individuais.

Considerando tal segmentação, analisar a relação entre acesso a planos e seguros de saúde e inserção no mercado de trabalho é de grande relevância tanto para a compreensão da dinâmica do mercado de trabalho como para a de acesso aos serviços de saúde no Brasil. A posse do plano de saúde altera a dinâmica do mercado de trabalho no que se refere à inserção e à mobilidade entre postos de trabalho, e essa relação está condicionada pelas preferências e atributos individuais. Por outro lado, o acesso aos serviços de saúde gera ganhos de bem-estar para os indivíduos e determina o estoque de capital humano individual, resultando em impactos nos fluxos de renda futuros. No Brasil, como a cobertura de plano de saúde é, em grande medida, associada ao vínculo empregatício, o entendimento da capacidade de expansão dessa cobertura torna premente a análise dessa relação.

No Brasil, são escassos os trabalhos que analisam a relação entre mercado de trabalho e acesso a planos de saúde. Maia et al. ${ }^{8}$ mostram que a cobertura de planos coletivos no Brasil tende a diminuir com a idade enquanto a cobertura por planos individuais tende a aumentar, associando esse comportamento à participação no mercado de trabalho. Os idosos têm uma taxa de participação menor e quando se aposentam, muitas vezes, perdem o direito de manter o mesmo benefício ao qual tinham acesso quando ativos. Além disso, apontam que a renda familiar per capita e a escolaridade apresentam uma relação monotônica estritamente crescente com a probabilidade de ter plano. No caso dos $10 \%$ mais ricos e com 
Ensino Superior completo, a chance é duas vezes maior do que do grupo dos $20 \%$ mais ricos e com Ensino Médio completo. Estar inserido no setor formal é outra condição relevante para acesso a plano de saúde coletivo. Desempregados e inativos apresentam maiores chances de adquirir planos individuais vis-à-vis empregados, algo explicado pela própria condição de atividade/ ocupação.

O objetivo deste trabalho é caracterizar a cobertura de planos privados de saúde segundo a inserção dos indivíduos no mercado de trabalho brasileiro. Os suplementos da Pesquisa Nacional de Amostra por Domicílios - PNAD (Instituto Brasileiro de Geografia e Estatística; http:/ /www. ibge.gov.br) de 1998 e 2003 informam sobre status de cobertura de plano privado de saúde ao mesmo tempo em que permitem identificar o arranjo ocupacional. Considerando essas informações, pretende-se definir grupos homogêneos de trabalhadores conforme atributos pessoais (sexo, raça, idade, escolaridade), características dos domicílios (referentes ao chefe, presença de idosos e presença de crianças, entre outras) e características do posto de trabalho (condição de atividade, categorias ocupacionais, rendimento, sindicalização) além de condições de saúde, acesso a serviços e tipos de planos.

A definição de uma tipologia é requerida por não contarmos, no Brasil, com informações de estabelecimento sobre a população que tem acesso a planos de saúde. Na verdade, não estão disponíveis dados administrativos que compreendam a população como um todo e possam com isso garantir uma análise refinada da relação entre mercado de trabalho e mercado de seguro saúde. Recorre-se, portanto, aos dados secundários de pesquisas domiciliares amostrais como a PNAD para construção de tipologia e posterior análise dessa relação. A amostra se restringe a seis regiões metropolitanas brasileiras, já que o acesso a plano de saúde é um fenômeno essencialmente urbano dado o grau de organização do mercado de trabalho nessas áreas.

Para construção da tipologia, recorre-se ao método Grade of Membership (GoM) - ou Grau de Pertencimento. Cinco perfis foram definidos, utilizando mais de vinte variáveis categóricas e, entre elas, as variáveis-chave para denominação dos grupos são: grau de cobertura de planos de saúde, condição de atividade e escolaridade do indivíduo. Entre os perfis, predominam as categorias de baixo grau de cobertura, o que reforça a necessidade de se formularem ações a fim de ampliar o acesso ao plano de saúde, não somente para melhorar o bem-estar, mas também para atender a uma população que vem envelhecendo em ritmo acelerado. Por outro lado, os resultados mostram que a categoria relacionada aos indivíduos ocupados com idade próxima de 30 anos é de alto grau de cobertura.

$\mathrm{O}$ artigo está dividido em seis seções, incluindo essa Introdução. A seguir, em Métodos, apresenta-se o método GoM de forma sumária. Em Fonte de Dados e Variáveis, faz-se uma breve revisão da literatura sobre a relação entre mercado de trabalho e mercado de seguro saúde. A análise descritiva das categorias tipológicas segundo variáveis selecionadas é feita nos Resultados e, por fim, na Discussão, tecem-se algumas considerações.

\section{Métodos}

O GoM é um método filiado à teoria dos conjuntos nebulosos, para a qual os elementos podem ter "graus" de pertencimento a um ou mais conjuntos, ao invés de, conforme estabelecido na teoria clássica de conjuntos, simplesmente pertencer ou não a determinado conjunto. Com base na distribuição de características entre os elementos da amostra investigada, o GoM estima, pelo método de máxima verossimilhança, os atributos prováveis dos perfis extremos e o grau de "proximidade" (ou associação) de cada elemento ${ }^{9}$. O número de perfis extremos (ou perfis de referência) deve ser estipulado a priori e definido conforme o conhecimento do pesquisador no assunto em questão, e resultará na dimensão final da tipologia, ou seja, no número total de categorias, que incluirá tipos puros e mistos. Os tipos mistos combinam características de dois ou mais tipos puros, os quais, por sua vez, correspondem basicamente aos perfis extremos.

Para cada elemento (nesse caso, indivíduo) em um conjunto nebuloso, existe um grau de pertinência $\left(\mathrm{g}_{i k}\right)$ que representa o grau com que o elemento $i$ pertence ao conjunto/perfil $k$, assumindo valores entre 0 (zero) e 1 (um). Se $g_{i k}$ é igual a 0 , o elemento não pertence ao conjunto. No caso de assumir valor igual a 1, o elemento pertence completamente ao conjunto. Quando $\mathrm{g}_{i k}$ assume valores maiores que 0 e menores que 1 , o indivíduo tem pertencimento (parcial) a mais de um perfil de referência 10.

Os escores $g_{i k}$ estão sujeitos às seguintes restrições:

$0 \leq \mathrm{g}_{i k} \leq 1$ para cada $i$ e cada $k$

$\Sigma \mathrm{g}_{i k}=1$ para cada $i$

O parâmetro lambda $\lambda$, importante para discriminar quais atributos estão relacionados a cada perfil de referência, é dado pela probabilidade de resposta do $j$-ésimo atributo pelo elemento com $k$-ésimo perfil extremo. $\lambda_{k j l}$ é dessa 
forma definida e deve atender às seguintes pressuposições:

$0 \leq \lambda_{k j l} \leq 1$ para cada $k$, j e $l$

$\Sigma \lambda_{k j l}=1$ para cada $k \mathrm{e} j$

$\operatorname{Pr}\left(\mathrm{Y}_{i j l}=1\right)=\Sigma \mathrm{g}_{i k} \lambda_{k j l}$

E a função de máxima verossimilhança a ser estimada é:

$\mathrm{L}(\mathrm{Y})=\prod_{i} \Pi_{j} \Pi_{l}\left(\Sigma \mathrm{g}_{i k} \lambda_{k j l}\right)$

No caso de o pesquisador escolher dois perfis extremos, por análise combinatória, serão gerados cinco perfis/categorias que são definidos como puros ou mistos, de acordo com os seus graus de pertencimento a cada um dos perfis extremos, conforme é apresentado abaixo.

1) O indivíduo $i$ é considerado como pertencente ao perfil puro $m$ quando tiver os seguintes graus de pertencimento $g$ aos perfis extremos $m$ e $n$ :

a) $\mathrm{g}_{\text {im }} \geq 0,75$; ou

b) $0,50<$ g $_{\text {im }}<0,75$, desde que $g_{\text {in }} \leq 0,25$.

2) O indivíduo $i$ é considerado como pertencente ao perfil puro $n$ quando tiver os seguintes graus de pertencimento $g$ aos perfis extremos $m$ e $n$ :

a) $\mathrm{g}_{\text {in }} \geq 0,75$; ou

b) $0,50<$ g $_{\text {in }}<0,75$, desde que $g_{\text {im }} \leq 0,25$

3) O indivíduo $i$ é considerado como pertencente ao perfil misto de $n$ com $m$ (em que predominam as características de $n$ ), aquele cujos graus de pertencimento aos perfis $n$ e $m$ são:

a) $0,50<\mathrm{g}_{\text {in }} \leq 0,75$, desde que $0,25 \leq \mathrm{g}_{\text {im }}<0,50$.

4) O indivíduo $i$ é considerado como pertencente ao perfil misto de $m$ com $n$ (em que predominam as características de $m$ ), aquele cujos graus de pertencimento aos perfis $m$ e $n$ são:

a) 0,50< gim $_{\text {im }} \leq 0,75$, desde que 0,25 $\leq$ gin $_{\text {in }}<0,50$.

5) O indivíduo i do tipo misto sem predomínio é aquele com os seguintes graus de pertencimento $g$ :

a) $\mathrm{g}_{\text {im }}<0,50$ e $\mathrm{g}_{\text {in }}<0,50$; ou

b) $\left(\mathrm{g}_{\text {im }}=0,50\right)$ ou $\left(\mathrm{g}_{\text {in }}=0,50\right)$.

Desse modo, os perfis extremos resultam da estimação por máxima verossimilhança, à medida que as categorias finais da tipologia, puras e mistas, são definidas conforme os limites dados pelo algoritmo acima, ou seja, pela intensidade do grau de pertencimento ou pela localização em relação a um dos perfis extremos. $O$ perfil misto 0 é aquele em que não há predomínio de nenhum dos perfis extremos.

\section{Fonte de dados e variáveis}

A fonte de dados utilizada é a PNAD e os suplementos dos anos de 1998 e 2003. Tal pesquisa traz informações sobre o domicílio, o indivíduo e, no caso específico desses anos, sobre as condições de saúde da população brasileira. Como o acesso a plano é uma realidade típica de áreas urba- nas, basicamente metropolitanas, optamos por construir a tipologia no âmbito de seis regiões metropolitanas: Recife (Pernambuco), Salvador (Bahia), Belo Horizonte (Minas Gerais), Rio de Janeiro, São Paulo e Porto Alegre (Rio Grande do Sul). Assim, a amostra é de 127.131 observações. Tal amostra se refere ao "empilhamento" das PNAD que trazem o suplemento especial de saúde (1998 e 2003) e não abarcam os mesmos domicílios, pois essa pesquisa domiciliar não é longitudinal.

As variáveis utilizadas são categorizadas por exigência do método GoM. Para o método, quanto maior o número de variáveis, melhor a descrição dos perfis e a estimação dos graus de pertencimento. Para indivíduos acima de 18 anos, foram selecionadas 33 variáveis (Tabela 1) referentes aos atributos do indivíduo, ao tamanho e à composição do domicílio, à área metropolitana, às condições individuais de saúde, às características de inserção no mercado de trabalho, rendimento do trabalho, de todas as fontes e rendimentos per capita. As variáveis de renda foram organizadas segundo distribuições por quintis, com os menores valores no primeiro quinto e assim por diante. A variável de saúde autoavaliada na PNAD apresenta cinco categorias: muito boa, boa, regular, ruim, muito ruim. Os indivíduos que responderam à autoavaliação do estado de saúde como muito boa e boa foram classificados como saudáveis e os demais como não saudáveis.

Os atributos individuais dizem respeito àqueles que interferem na posição no mercado de trabalho e também na facilidade ou não de possuir um plano de saúde, já discutidos na seção anterior. São elas: sexo, idade, raça, escolaridade. Em relação a tamanho e composição do domicílio, selecionamos número de filhos em faixas etárias diferentes, presença de idoso e características do chefe. A composição do domicílio pode tornar a necessidade do plano de saúde mais premente, pois quanto maior a idade dos moradores ou o número de crianças, maior tende a ser a demanda por serviços de saúde. As informações sobre gastos com saúde não foram incluídas por estarem disponíveis apenas no suplemento da PNAD de 1998. Além disso, cabe ressaltar que a qualidade das informações de gasto com saúde de forma geral parece não ter sido bem aferida na PNAD, particularmente pela dificuldade de diferenciar se os gastos declarados são individuais ou familiares. No caso específico de plano de saúde, como nem sempre é o titular do plano que responde ao questionário da PNAD, essas informações se mostraram pouco consistentes ${ }^{11}$.

No que tange às características do mercado de trabalho, são informações relevantes: condição de atividade, posição na ocupação (deno- 
Perfis extremos conforme maiores probabilidades de respostas às variáveis.

\begin{tabular}{|c|c|c|}
\hline Variável & Perfil 1 & Perfil 2 \\
\hline Ano & 1998 & Não discrimina \\
\hline Sexo & Não discrimina & Não discrimina \\
\hline Raça & Não discrimina & Não discrimina \\
\hline Contribuinte de instituto de previdência & Não discrimina & $\operatorname{Sim}$ \\
\hline Escolaridade do morador & 0 a 3 anos e 4 a 7 anos & 8 a 10 anos, 11 a 14 anos e 15 anos ou mais \\
\hline Idade do morador & 54 a 58 anos e 59 anos ou mais & 24 a 33 anos, 34 a 38 anos e 44 a 48 anos \\
\hline Escolaridade do chefe & 0 a 3 anos & 8 a 10 anos, 11 a 14 anos e 15 anos ou mais \\
\hline Economicamente ativa & Não & Sim \\
\hline Renda familiar per capita & 1으, 2으 e 3ㅇ quintis & 4o e 5 o quintis \\
\hline Saúde autoavaliada & Não & Sim \\
\hline Tem plano de saúde & Não & $\operatorname{Sim}$ \\
\hline Tipo de plano de saúde & Missing & Público, privado e voluntário \\
\hline Dependentes no plano & Não discrimina & 2 a 4 dependentes \\
\hline Cobertura de plano & Missing & Hospitalar \\
\hline Número de filhos na família com até 1 ano de idade & 2 a 4 filhos e 5 filhos ou mais & 1 filho \\
\hline Número de filhos na família de 2 a 7 anos de idade & Nenhum filho & 1 filho e 2 a 4 filhos \\
\hline Número de filhos na família de 8 a 15 anos de idade & Não discrimina & Não discrimina \\
\hline Aposentado & Sim & Não discrimina \\
\hline Pensionista & Sim & Não discrimina \\
\hline Presença de crianças com até 10 anos de idade & Não & Não discrimina \\
\hline Presença de idosos a partir de 65 anos de idade & Não & Não discrimina \\
\hline Idade do chefe & 19 a 23 anos e 59 anos ou mais & 44 a 48 anos e 49 a 53 anos \\
\hline Região metropolitana & Recife (Pernambuco) & São Paulo \\
\hline Número de membros & 5 membros ou mais & Não discrimina \\
\hline Tempo no trabalho & Missing e mais de um ano & Menos de um ano \\
\hline Composta & Inativo e procurando & Doméstica, informal e formal \\
\hline Skill & Missing & Superior, médio e manual \\
\hline Status marital & Casado & Não discrimina \\
\hline Rendimentos não trabalho & 1 으 e 2 o quintis & Não discrimina \\
\hline Log (rendimentos não trabalho) & 2o quintil & 5ㅇ quintil \\
\hline Sindicalizado & Missing & Sim e não \\
\hline Salário-hora do trabalho principal & Missing e 1ㅇq quintil & 3 으, 4 으 e 5 ㅇ quintis \\
\hline Renda de todos os trabalhos & Missing, 1으 e 2o quintis & 3으, 4 으 e 5 으 quintis \\
\hline
\end{tabular}

Fonte: elaboração própria com base na Pesquisa Nacional por Amostra de Domicílios - PNAD 1998 e 2003 (Instituto Brasileiro de Geografia e Estatística; http://www.ibge.gov.br).

minada aqui variável composta), contribuição para a Previdência, sindicalização, tempo de não trabalho e categoria ocupacional (skill) que trata de ocupações que requerem habilidades de nível superior, de médio ou são consideradas manuais. Tais variáveis são, na verdade, indicativas de quão precária ou quão pouco formalizada é a participação no mercado de trabalho, sugerindo a dificuldade de cobertura do plano de saúde.

Os perfis extremos foram definidos pela análise entre a razão de probabilidades a cada resposta da variável empregada (lambda) e a frequência marginal das respostas. Os perfis foram identificados segundo o critério de corte proposto por Melo 9: a variável discrimina ou importa se a razão entre o lambda e a frequência marginal de cada resposta for superior a 1,2. Vale dizer, sempre que, em um certo perfil de referência, a probabilidade de determinada resposta a uma variável superar em $20 \%$ a frequência dessa resposta na amostra, considera-se que a característica alcança alta probabilidade no perfil e, portanto, o discrimina. Isso não significa que o perfil extremo engloba todos os indivíduos com 
as características nele prevalecentes, mas sim que há maior chance de isso ocorrer. Ressalte-se, ainda, que a descrição inicial dos perfis baseada nas probabilidades é, posteriormente, checada e corroborada (ou não) pela distribuição das características entre os tipos puros e mistos.

\section{Resultados}

A Tabela 1 traz a descrição das características predominantes dos perfis extremos definidos pelo método GoM.

A aplicação do algoritmo de graus de pertencimento aos perfis extremos acima possibilitou a definição de cinco categorias em uma tipologia que envolve condição de atividade/ocupação, acesso a planos de saúde e escolaridade. As categorias definidas e a respectiva participação relativa são: "inativos sem acesso a plano com 0 a 3 anos de estudo" (29,52\%); "inativos e desempregados, com relativo acesso a plano com 0 a 3 e mais de 11 anos de estudo" (17,96\%), "ocupados, menor acesso a plano, 4 a 7 anos de estudo" $(15,44 \%)$ e "ocupados, maior acesso a plano e com mais de 11 anos de estudo" (37,07\%).

Buscando caracterizar o perfil dessas categorias de acordo com as variáveis utilizadas para definição da tipologia, analisam-se a composição de cada grupo segundo os atributos de área metropolitana de residência, o acesso a plano, a condição de atividade e a escolaridade. A seguir, as categorias são tipificadas por características individuais como sexo, idade, percepção da saúde, condição de atividade/ocupação, sindicalização e rendimento do trabalho. Essa caracterização nos permite verificar em que medida a tipologia definida pelo GoM cria grupos realmente homogêneos segundo alguns atributos. A Tabela 2 apresenta os resultados dessa caracterização.

Em Recife, predomina a categoria de "inativos, sem acesso a plano, com 0 a 3 anos de estudo", uma vez que a participação dela é superior à média na metrópole. Essa categoria está, entre as cinco, na pior condição por conta da inatividade combinada ao baixo nível de escolaridade. Tal resultado é esperado, haja vista o grau de desenvolvimento socioeconômico das metrópoles nordestinas frente às do Sudeste e Sul. "Inativos e desempregados, com relativo acesso a plano com 0 a 3 e mais de 11 anos de estudo" localizamse, em maior medida, em São Paulo. Em Salvador, concentram-se os indivíduos de 0 a 3 anos de estudo e, em São Paulo, os com mais de 11 anos de estudo. A categoria "ocupados, menor acesso a plano, 4 a 7 anos de estudo" se localiza, com participação relativamente acima da média, em Porto Alegre. Finalmente, “ocupados, maior acesso a plano, mais de 11 anos de estudo" é uma categoria sobrerrepresentada em São Paulo e Porto Alegre. Trata-se, seguramente, da categoria de melhor inserção socioeconômica e, não é por mero acaso, que apresenta participações mais elevadas nessas duas metrópoles.

Em relação a acesso a planos de saúde, a categoria em que há maior cobertura são os "ocupados com mais de 11 anos de estudo" que apresenta cerca de $60 \%$ dos indivíduos com acesso a planos de saúde. A categoria com menos expressão nesse quesito é "inativos sem acesso a plano, 0 a 3 anos de estudo" na qual apenas $8 \%$ dos indivíduos têm acesso a algum tipo de plano de saúde. Essa enorme diferença reforça que o acesso a plano ocorre em segmentos muito específicos do mercado de trabalho, particularmente para trabalhadores mais qualificados.

A segunda variável-chave na definição da tipologia é a condição de atividade que apresenta as categorias de empregados, desempregados e inativos. Nas categorias "inativos sem acesso a plano, 0 a 3 anos de estudo" e "inativos e desempregados, com relativo acesso a plano, 0 a 3 e mais de 11 anos de estudo" tem-se 74,52\% e $50,64 \%$ de inativos, respectivamente. A categoria que apresenta maior percentual de pessoas ocupadas associadas à população economicamente ativa é a de "ocupados, maior acesso a plano, mais de 11 anos de estudo", quase 100\%.

A análise das categorias segundo anos de estudo revela também grandes disparidades entre os grupos. A categoria que ganha a denominação de "inativos, sem acesso a plano, 0 a 3 anos de estudo" é aquela cuja participação de indivíduos com tal nível de escolaridade é de $36 \%$, bem superior à média da amostra (18,51\%). Não é desprezível a participação nesta categoria dos indivíduos que possuem de 4 a 7 anos, quase $40 \%$, porém a distância é menor relativamente à média $(29,65 \%)$. Por outro lado, o perfil “ocupados com maior acesso a plano, com mais de 11 anos de estudo" detém 42,44\% dos indivíduos com escolaridade entre 11 a 14 anos e $19,03 \%$ com mais de 15 anos.

No que concerne aos atributos individuais, a composição por sexo mostra predomínio das mulheres nos grupos com maior participação de “inativos”, ao passo que a distribuição é mais uniforme nas de ocupados, com ligeiro predomínio dos homens.

A distribuição dos grupos por faixa etária mostra padrões interessantes. Os perfis "inativos” apresentam predominância relativa na faixa com mais de 59 anos de idade, conforme esperado, enquanto os grupos de "ocupados" apresentam dois comportamentos. A categoria “ocupados, menor acesso a plano, 4 a 7 anos de 
Distribuição dos atributos segundo as categorias definidas na tipologia.

\begin{tabular}{|c|c|c|c|c|c|c|}
\hline \multirow[t]{2}{*}{ Composição das categorias (\%) } & \multicolumn{5}{|c|}{ Perfis } & \multirow[t]{2}{*}{ Média } \\
\hline & 0 & 1 & 2 & 3 & 4 & \\
\hline \multicolumn{7}{|l|}{ Região Metropolitana (Estado) } \\
\hline Recife (Pernambuco) & 21,43 & 22,05 & 17,97 & 14,71 & 9,70 & 17,17 \\
\hline Salvador (Bahia) & 7,14 & 15,27 & 15,32 & 14,76 & 11,45 & 12,79 \\
\hline Belo Horizonte (Minas Gerais) & 35,71 & 12,34 & 13,21 & 14,83 & 14,15 & 18,05 \\
\hline Rio de Janeiro & 21,43 & 18,36 & 17,98 & 18,13 & 18,26 & 18,83 \\
\hline São Paulo & 7,14 & 17,59 & 20,07 & 19,46 & 24,90 & 17,83 \\
\hline Porto Alegre (Rio Grande do Sul) & 7,14 & 14,39 & 15,44 & 18,10 & 21,54 & 15,32 \\
\hline \multicolumn{7}{|l|}{ Acesso ao plano } \\
\hline Missing & 0,00 & 0,02 & 0,00 & 0,02 & 0,01 & 0,01 \\
\hline Sim & 28,57 & 8,00 & 49,92 & 27,12 & 59,50 & 34,02 \\
\hline Não & 71,43 & 91,99 & 53,08 & 72,86 & 40,49 & 65,97 \\
\hline \multicolumn{7}{|l|}{ Condição de atividade } \\
\hline Ocupados & 64,29 & 7,03 & 36,35 & 81,81 & 99,98 & 57,89 \\
\hline Desempregados & 7,14 & 18,45 & 13,01 & 6,71 & 0,02 & 9,07 \\
\hline Inativos & 28,57 & 74,52 & 50,64 & 11,48 & 0,00 & 33,04 \\
\hline \multicolumn{7}{|l|}{ Anos de estudo } \\
\hline Missing & 0,00 & 0,41 & 0,40 & 0,46 & 0,57 & 0,37 \\
\hline $0-3$ & 14,29 & 36,52 & 20,77 & 19,18 & 1,81 & 18,51 \\
\hline $4-7$ & 28,57 & 39,08 & 27,68 & 35,48 & 17,41 & 29,65 \\
\hline $8-10$ & 21,43 & 16,33 & 15,47 & 15,85 & 18,74 & 17,56 \\
\hline $11-14$ & 28,57 & 7,25 & 29,49 & 23,39 & 42,44 & 26,23 \\
\hline Mais de 15 & 7,14 & 0,39 & 6,19 & 5,64 & 19,03 & 7,68 \\
\hline \multicolumn{7}{|l|}{ Sexo } \\
\hline Feminino & 35,71 & 68,80 & 62,48 & 46,13 & 44,24 & 48,53 \\
\hline Masculino & 64,29 & 31,20 & 37,52 & 53,87 & 55,76 & 51,47 \\
\hline \multicolumn{7}{|l|}{ Faixa etária (anos) } \\
\hline Missing & 0,00 & 0,03 & 0,03 & 0,03 & 0,02 & 0,02 \\
\hline $19-23$ & 21,43 & 14,51 & 15,01 & 15,80 & 13,96 & 16,14 \\
\hline $24-33$ & 28,57 & 19,08 & 20,13 & 25,21 & 30,22 & 24,64 \\
\hline $34-38$ & 14,29 & 8,95 & 10,19 & 11,89 & 14,69 & 12,00 \\
\hline $39-43$ & 0,00 & 7,65 & 8,87 & 12,35 & 14,17 & 8,61 \\
\hline $44-48$ & 7,14 & 6,36 & 7,50 & 11,15 & 12,27 & 8,88 \\
\hline $49-53$ & 28,57 & 6,70 & 7,79 & 9,31 & 8,22 & 12,12 \\
\hline $54-58$ & 0,00 & 6,77 & 7,22 & 6,08 & 4,01 & 4,82 \\
\hline Mais de 59 & 0,00 & 29,95 & 23,26 & 8,18 & 2,44 & 12,77 \\
\hline \multicolumn{7}{|l|}{ Autoavaliação da saúde } \\
\hline Missing & 0,00 & 0,02 & 0,01 & 0,02 & 0,02 & 0,01 \\
\hline Saudável & 92,86 & 56,84 & 70,47 & 76,82 & 90,23 & 77,44 \\
\hline Não saudável & 7,14 & 43,14 & 29,52 & 23,16 & 9,75 & 22,54 \\
\hline \multicolumn{7}{|l|}{ Ocupação } \\
\hline Missing & 0,00 & 1,99 & 2,47 & 2,70 & 0,46 & 1,52 \\
\hline Inativo & 28,57 & 74,52 & 50,64 & 11,48 & 0,00 & 33,04 \\
\hline Desempregado & 7,14 & 18,45 & 13,01 & 6,71 & 0,02 & 9,07 \\
\hline Doméstica & 0,00 & 1,06 & 8,11 & 12,56 & 3,79 & 5,1 \\
\hline Informal & 42,86 & 3,87 & 19,31 & 36,19 & 26,25 & 25,70 \\
\hline Formal & 21,43 & 0,11 & 6,47 & 30,35 & 69,48 & 25,57 \\
\hline
\end{tabular}

(continua) 


\begin{tabular}{|c|c|c|c|c|c|c|}
\hline \multirow[t]{2}{*}{ Composição das categorias (\%) } & \multicolumn{5}{|c|}{ Perfis } & \multirow[t]{2}{*}{ Média } \\
\hline & 0 & 1 & 2 & 3 & 4 & \\
\hline \multicolumn{7}{|l|}{ Sindicalização } \\
\hline Missing & 28,57 & 79,20 & 53,66 & 12,37 & 0,00 & 34,76 \\
\hline Sim & 7,14 & 0,89 & 3,42 & 9,92 & 26,46 & 9,57 \\
\hline Não & 64,29 & 19,91 & 42,92 & 77,71 & 73,54 & 55,67 \\
\hline \multicolumn{7}{|l|}{$\begin{array}{l}\text { Rendimento hora-médio-real } \\
\text { (trabalho) }\end{array}$} \\
\hline Missing & 42,86 & 48,67 & 35,81 & 12,86 & 1,40 & 28,32 \\
\hline 1ㅇ quintil & 7,14 & 49,22 & 35,22 & 13,52 & 0,63 & 21,15 \\
\hline 2o quintil & 14,29 & 1,73 & 8,38 & 10,75 & 3,19 & 7,67 \\
\hline 3o quintil & 14,29 & 0,12 & 4,24 & 13,59 & 11,12 & 8,67 \\
\hline $4^{\circ} \circ$ quintil & 0,00 & 0,22 & 10,85 & 25,08 & 22,77 & 11,78 \\
\hline 5o quintil & 21,43 & 0,05 & 5,50 & 24,20 & 60,89 & 22,41 \\
\hline Média & 0,01 & 29,52 & 17,96 & 15,44 & 34,07 & \\
\hline
\end{tabular}

Perfis: 0 (sem predomínio); 1 (inativos, sem acesso ao plano, 0 a 3 anos de estudo); 2 (inativos e desempregados, relativo acesso a plano, 0 a 3 ou mais de 11 anos de estudo); 3 (ocupados, menor acesso a plano, 4 a 7 anos de estudo) e; 4 (ocupados, maior acesso a plano, mais de 11 anos de estudo).

estudo" está concentrada nas faixas etárias de 39 a 43 anos e 44 a 48 anos, uma vez que essas participações são superiores às participações médias desses grupos etários, 8,61\% para a faixa de 39 a 43 anos e 8,88\% para a faixa de 44 a 48 anos. $\mathrm{O}$ segundo perfil de ocupados - "ocupados, maior acesso a plano, com mais de 11 anos de estudo" é predominantemente jovem, 30,22\% com idade entre 24 e 33 anos. Esse perfil de comportamento evidencia o efeito coorte associado à educação no Brasil.

Em todos os perfis, a autopercepção sobre as condições de saúde é boa. Mais de $70 \%$ declaram que a saúde é boa, com exceção do perfil "inativos, sem acesso a plano, 0 a 3 anos de estudo", no qual pouco mais da metade declara que é boa. Certamente, essa avaliação está associada à composição etária, porque, como já visto, há relativa incidência de pessoas mais idosas nessa categoria.

Quanto à composição por atividade e ocupação, ressalta-se a categoria "ocupados, maior acesso a plano, mais de 11 anos de estudo" por ser fortemente composta de trabalhadores formais $(69,48 \%)$. O outro perfil relativo aos ocupados se distribui uniformemente entre formal e informal, além de apresentar participação de domésticas superior a da mesma posição na amostra.

Do ponto de vista da cobertura sindical, há expressiva participação de missing nos perfis de “inativos", porque só respondem a essa questão aqueles que estão trabalhando. Em todas as ca- tegorias de "ocupados" ocorre baixa participação de sindicalizados. Entretanto, na de "ocupados, maior acesso a plano, com mais de 11 anos de estudo", em que a formalização das relações de trabalho é mais evidente, há maior representação de trabalhadores sindicalizados $(26,46 \%)$. Conforme ressaltado na literatura, em especial Gruber 6, há uma forte associação entre acesso a planos de saúde e sindicalização dos trabalhadores. Porém, tal resultado não parece ser robusto para o caso brasileiro.

Por fim, são analisados os grupos segundo a distribuição por quintos do rendimento-hora médio real do trabalho corrigido pelo deflator IPC construído especialmente para deflacionar a PNAD. O deflator é disponibilizado na página eletrônica: http://www.ipeadata.gov.br. Claramente, percebe-se maior participação relativa de "ocupados, maior acesso a plano, mais de 11 anos de estudo" entre os $20 \%$ mais ricos. De outra maneira, "inativos e desempregados, relativo acesso a plano, 0 a 3 anos ou mais de 11 anos" concentra-se no primeiro e segundo quintos da distribuição do rendimento do trabalho. Na categoria "ocupados, menor acesso a plano, 4 a 7 anos de estudo", os indivíduos se localizam no quarto quintil da distribuição do rendimentohora real do trabalho. 


\section{Discussão}

Este artigo constrói e descreve uma tipologia ocupacional com base nas PNAD de 1998 e 2003 que apresentam a vantagem de trazer informações sobre saúde dos indivíduos no suplemento especial. Os achados na análise descritiva, de um modo geral, corroboram as evidências apresentadas na literatura nacional e internacional. Entre eles, destaca-se a relação positiva entre cobertura de plano, rendimento do trabalho e, em alguma medida, sindicalização. Tal relação sugere o papel que as grandes empresas, sejam elas públicas ou privadas, detêm na criação de um mercado de trabalho mais "protegido", contribuindo colateralmente para fomentar o mercado de planos coletivos privados. A análise dos tipos evidencia a natureza segmentada do mercado de trabalho brasileiro. Os grupos 3 e 4, que são constituídos por ocupados, refletem a dualidade do mercado de trabalho brasileiro. No primeiro grupo, temos trabalhadores mais formalizados, com maior nível de escolaridade e rendimentos, como também associados a algum sindicato. Entretanto, o grupo 4 é composto por trabalhadores informais e empregados domésticos, que apresentam menor nível de escolaridade e auferem rendimentos mais baixos. Nesse contexto, o acesso a plano de saúde é mais uma variável que contribui para a agudização dessa segmentação e altera a dinâmica do mercado de trabalho. Os trabalhadores ocupados e mais escolarizados são os que possuem maior acesso a plano de saúde, o que caracteriza os postos de trabalho desse perfil de trabalhadores como de maior qualidade. $\mathrm{Na}$ perspectiva de longo prazo, esses trabalhadores com maior acesso a plano de saúde têm maiores condições de acumular capital humano, reiterando um equilíbrio perverso em que coexistem dois grupos com baixa possibilidade de mobilidade entre eles.

Por outro lado, o acesso ou não a planos de saúde estabelece forte associação a dois determinantes próximos: renda e idade. E a renda é basicamente influenciada pela condição de atividade/ocupação e nível de escolaridade. Esses dois determinantes - renda e idade - frequentemente entram em contradição. Por exemplo, no caso dos mais velhos, em razão das piores condições de saúde, a fase do ciclo de vida em que se encontram contribuiria para a filiação a algum plano. Todavia, dois aspectos negativos se combinam. Por um lado, a renda não é suficiente por serem inativos e por, em sua maioria, deterem menor nível de escolaridade. Por outro lado, por serem mais velhos, estão sujeitos a cobranças de prêmios de seguro mais elevados. Nos dois primeiros grupos, constituídos em sua maioria por inativos, observa-se grau de cobertura relativamente baixo comparado ao grupo dos ocupados. No Brasil, o benefício de acesso a plano de saúde nem sempre é garantido quando os indivíduos se aposentam, quando se trata da etapa do ciclo de vida na qual os indivíduos mais necessitam dos serviços de saúde.

Essas evidências nos remetem à necessidade de formulação de políticas que incentivem as empresas a ofertar o benefício do acesso ao plano de saúde. A Constituição Federal de 1988 prevê a possibilidade de acesso aos serviços de saúde suplementar, configurando o sistema de saúde brasileiro como um sistema misto em que é permitida a dupla entrada no sistema. O melhor instrumento para viabilizar tal acesso é o vínculo empregatício, e no Brasil isso ocorre tanto para os trabalhadores do setor privado quanto para os do setor público. Contudo, no setor privado, no qual há maior prevalência de vínculos precários, essas oportunidades de acesso não são uniformemente distribuídas, o que justificaria intervenções públicas para minimizar essa desigualdade. Apesar de a duplicidade de acesso ser inequitativa, a expansão do setor de saúde suplementar pode caracterizar uma maior disponibilidade de recursos em saúde pública para a população que está fora do mercado de trabalho e, portanto, mais vulneráveis, como inativos e desempregados.

Considerando a forma de rateio do financiamento do plano de saúde entre empresas e empregados, mesmo que as empresas assumam maior participação, a decisão das empresas em ofertar plano de saúde será orientada por uma relação de custo-benefício. Nos segmentos de mercado de trabalho em que experiência não é elemento importante, esse benefício não existirá. Por essa razão, esses incentivos deveriam ser focalizados nas empresas que recrutam trabalhadores menos qualificados, pois tal medida, além de aumentar o bem-estar do trabalhador e de seus dependentes, tende a elevar a produtividade das empresas, inibindo a elevada rotatividade e assegurando mecanismos de proteção social quando da entrada na aposentadoria.

Já que a tipologia aqui elaborada foi capaz de identificar a expressiva relação entre o acesso a plano de saúde e as condições de inserção no mercado de trabalho, a intenção de maior formalização do mercado de trabalho brasileiro, presente no discurso dos gestores dessa área, deve ter em conta oportunidades de ampliação dos planos de saúde privados, pois a posse deles pode garantir maior estabilidade no posto de trabalho. Tal tipo de proteção assegura ganhos de experiência e incentivos para capacitação do trabalhador, reduz o custo de intermediação de 
mão de obra e os dispêndios com seguro desemprego, contribuindo para aumento do bem-estar do trabalhador, para maior produtividade da em- presa e redução nos gastos governamentais com saúde e recolocação da mão de obra desempregada ou pouco qualificada.

\section{Resumo}

O objetivo deste trabalho é caracterizar a cobertura de planos privados de saúde segundo a inserção dos indivíduos no mercado de trabalho brasileiro. Considerando as informações sobre status de cobertura de plano de saúde e inserção no mercado de trabalho, foram definidos grupos homogêneos de trabalhadores. Para construção da tipologia, recorre-se ao método Grade of Membership (GoM) - ou Grau de Pertencimento, utilizando a Pesquisa Nacional de Amostra de Domicílios (PNAD) referente aos anos de 1998 e 2003, que apresentam suplemento de saúde. Cinco perfis de indivíduos foram definidos. As variáveis-chave para denominação dos grupos são: grau de cobertura de planos de saúde, condição de atividade e escolaridade do indivíduo. Os achados revelam relação positiva entre cobertura de plano, rendimento do trabalho e, em alguma medida, sindicalização.

Planos de Pré-Pagamento em Saúde; Cobertura de Serviços Privados de Saúde; Mercado de Trabalho

\section{Colaboradores}

A. F. Machado elaborou a resenha bibliográfica, fez a estimação pelo método GoM, analisou os resultados e aprovou a versão final do artigo. M. V. Andrade trabalhou na análise dos dados e interpretação dos resultados, na edição e aprovação da versão final do artigo. A. C. Maia organizou o banco de dados, incluindo a construção e crítica das variáveis utilizadas referentes à saúde e mercado de trabalho e aprovou a versão final do artigo. 


\section{Referências}

1. McDonald IM, Solow RM. Wage bargaining and employment. Am Econ Rev 1981; 71:896-908.

2. Akerlof, GA. Labor contract as partial gift exchange. Q J Econ 1982; 97:543-69.

3. Akerlof GA, Yellen J. Efficiency wages of the labor market. London: Cambridge University Press; 1986.

4. Weiss A. Job queues and layoffs in labor markets with flexible wages. J Polit Econ 1980; 88:526-38.

5. Cooper PF, Monheit AC. Does employment-related health insurance inhibit job mobility? Inquiry 1993; 30:400-16.

6. Gruber J. Health insurance and labor market. Cambridge: National Bureau of Economic Research; 1998. (NBER Working Papers Series, 6762).

7. Baicker K, Chandra A. The labor market effects of rising health insurance premiums. Cambridge: National Bureau of Economic Research; 2005. (NBER Working Papers Series, 11160).
8. Maia AC, Andrade MV, Ribeiro MM, Brito RJA. Estudo sobre a regulação do setor brasileiro de planos de saúde. Brasília: Secretaria de Acompanhamento Econômico, Ministério da Fazenda; 2006. (SEAE/MF Documento de Trabalho, 37).

9. Melo F. Trajetórias no mercado de trabalho: perfis socioocupacionais de indivíduos e casais da Grande São Paulo [Tese de Doutorado]. Belo Horizonte: Centro de Desenvolvimento e Planejamento Regional, Universidade Federal de Minas Gerais; 2006.

10. Manton KG, Woodbury MA, Tolley HD. Statistical applications using fuzzy sets. New York: John Wiley \& Sons; 1994.

11. Andrade MV. A saúde na PNAD. Belo Horizonte: Centro de Desenvolvimento e Planejamento Regional, Universidade Federal de Minas Gerais; 2002. (Texto para Discussão, 170).

Recebido em 20/Out/2010

Versão final reapresentada em 18/Out/2011

Aprovado em 23/Nov/2011 